\title{
Retractions of DOI: 10.1089/neu.2008.0707 and 10.1089/neu.2011.1842
}

Journal of Neurotrauma has been notified by the Acting Director of the Office of Research Integrity (ORI) of two published articles in the Journal where the authors engaged in research misconduct by falsifying and/or fabricating data by falsely reporting the results of Western blot experiments that examined neuroinflammation, amyloidogenesis, and/or congntive impairment in a rat model of cerebral ischemia. Specifically, the authors duplicated, reused, and falsely relabeled Western blot gel images and claimed they represented different experiments in a number of published articles in the scientific literature.

Acting in compliance with ORI, and in the interest of upholding the strictest standards of scientific publishing, the editorial leadership of Journal of Neurotrauma agrees that the research misconduct is of such significance as to warrant retraction of the following articles:

- Briones TL, Rogozinska M, Woods J. Environmental experience modulates ischemia-induced amyloidogenesis and enhances functional recovery. J Neurotrauma 2009;26(4): 613-625.

- Briones TL, Rogozinska M, Woods J. Modulation of ischemia-induced NMDAR1 activation by environmental enrichment decreases oxidative damage. J Neurotrauma 2011;28(12):2485-2492. 